\title{
One per Second Times Kilopascal
}

National Cancer Institute

\section{Source}

National Cancer Institute. One per Second Times Kilopascal. NCI Thesaurus. Code C127804.

A unit of resistance equal to the inverse of one kilopascal multiplied by a unit of time equal to one second. 